\title{
Effects of Lidocaine Spray on Pain and Anxiety in Patients Administered a Contrast Medium during Vein Puncture
}

\author{
Young-Mi $\mathrm{Oh}^{1}$ and Hyeon-Cheol Jeong ${ }^{2 *}$ \\ ${ }^{1}$ Deptartment of Nursing, Samhyook University Graduate School \\ ${ }^{2}$ Deptartment of Nursing, Samhyook University \\ loym0516@hanmail.net, \\ ${ }^{2 *}$ Corresponding
}

\begin{abstract}
This randomized controlled study with a comparative experimental design investigated the effects of using lidocaine spray with regard to the pain and anxiety of patients administered a contrast medium during venipuncture. Data were collected from 55 patients who visited the Department of Radiology at $N$ General Hospital, Seoul, for undergoing computed tomography or magnetic resonance imaging, met the subjectselection criteria, and provided consent for participating in the study during February and March 2015. The visual analog scale (VAS) was used to measure subjective pain, and the objective pain behavior checklist developed by Park was used to measure objective pain. Subjective anxiety was measured using the VAS, and the anxiety state was measured using the anxiety scale revised and supplemented by Leek. The study found that the experimental group sprayed with lidocaine during venipuncture scored significantly lower points for subjective pain $(t=-2.86, p=.006)$, objective pain $(t=-3.82, p<.001)$, subjective anxiety $(F=4.36, p=.042)$, and anxiety state $(F=5.02, p=.028)$ than did the control group. These findings indicate that lidocaine spraying influences pain reduction.
\end{abstract}

Keywords: Satisfaction, Lidocaine, Venipuncture, Pain, Anxiety

\section{Introduction}

With the improvements in medical care, cutting-edge medical equipment is being increasingly used. Computed tomography (CT) and magnetic resonance imaging (MRI) have greatly facilitated early cancer detection, management, and treatment. A contrast medium is used to distinguish tissues and blood vessels on CT and MRI images for obtaining accurate diagnostic information. Contrast media are watersoluble preparations containing an iodophor compound and provide contrast enhancement throughout the body including blood vessels. Further, the pattern of contrast enhancement can provide diagnostic pathological information [1]. CT and MRI require the intravenous injection of contrast medium via a catheter. Intravenous injection is a common nursing practice in medical organizations, which is used to supply drugs, fluids, blood, and nourishment [2-3]. Many patients have a mental resistance to and fear of the pain caused by intravenous injections [4].

Factors influencing pain due to injection include the type of syringe needle, practitioner's skill, number of injection attempts, and injection speed [5]. Previous studies have measured the pain caused by syringe needle insertion by using the 0 - to $10-\mathrm{cm}$ visual analogue scale (VAS). These studies have shown that adult vaccination injection pain was 21.3 points [6], anticancer drug intravenous injection pain was 37.9 points, and the pain experienced by patients with arteriovenous aneurysms undergoing blood dialysis was 49.0 points [5].

Venipuncture to inject a contrast medium for CT and MRI is a basic nursing skill. The needle $\left(18 \mathrm{G}^{\sim} 20 \mathrm{G}\right)$ used for venipuncture makes many patients feel pain and 
uneasiness. In the clinical field, nurses should not overlook the complaints of pain by patients undergoing venipuncture. To efficiently fulfill their role as care providers, nurses must adopt a multilateral and holistic approach to satisfy an individual patient's nursing needs [7]; effective and safe nursing intervention must be in place to ease the patient's pain.

Mental anxiety while experiencing or anticipating pain elevates the level of perceived pain. Moreover, the feeling of pain is more serious under anxiety or muscular tension [8]. Even normal people experience anxiety when risk or pain is expected or when unexpected events take place [9]. Anxiety is a negative mental reaction caused by stress or threatening situations. It is an important human experience closely related to human health, as well as a nursing problem requiring proper intervention [10]. Easing pain and anxiety is a major nursing intervention and requires nurses to gain an understanding of what their patients feel and to communicate with them [11]. Therefore, helping in reduction of pain and anxiety felt by patients during venipuncture for CT and MRI contrast media injection is an important mission for nurses.

Diverse therapies are used to lower the pain and anxiety caused by puncture procedures and biopsies, including non-invasive interventions such as the application of local analgesic ointments like the Eutectic Mixture of Local Anesthetics (EMLA) cream [12], music therapy [13], cold and warmth therapy [14], and aromatherapy [15]. These intervention methods have a lower risk of side effects and cause less anxiety compared to invasive measures [5]. Of these, the EMLA cream, albeit used as a non-invasive measure, has to be applied at least 1 hour prior to injection and taped upon, thereby imposing a time and cost burden [16]; therefore, it may not be suitable for anesthetizing the skin surface for venipuncture for CT and MRI contrast medium injection. In such situations, spraying lidocaine, a local anesthetic, can be an alternative approach for reducing the waiting time of patients undergoing CT and MRI.

Lidocaine spray is used for surface anesthesia. It is non-invasive and convenient to use and has been used for acute throat discomfort [17], paroxysmal pain in trigeminal neuralgia [18], intubation of organs [19], etc. In such cases, lidocaine was mostly applied on the skin surface, and this method has proven effective in many studies. However, to our knowledge, no study has examined the anesthetic effect of a surface anesthetic in venipuncture for contrast medium injection. The present study aimed to understand the effect of lidocaine spray on venipuncture pain and anxiety in patients undergoing contrast medium injection for CT and MRI and to explore the clinical application of lidocaine spray for reducing the pain and anxiety of patients, thereby improving the quality of nursing service.

\section{Study Method}

\subsection{Study Design}

This was a randomized, controlled, before-and-after study (Figure 1).

\begin{tabular}{cccc} 
Group & Pre-test & Intervention & Post-test \\
\hline Experimental & $\mathrm{E}_{1}$ & $\mathrm{X}_{1}$ & $\mathrm{E}_{2}$ \\
Control & $\mathrm{C}_{1}$ & $\mathrm{X}_{2}$ & $\mathrm{C}_{2}$ \\
\hline
\end{tabular}

$\mathrm{X}_{1}, 10 \%$ lidocaine; $\mathrm{X}_{2}$, normal saline

Figure 1. Research Design 


\subsection{Study Subjects}

This study included outpatients who had visited the general hospital N in Seoul, ROK, to receive venipuncture for CT or MRI. Of them, 30 patients who filled the written consent form to participate in the research experiment were randomly assigned to each group.

\subsection{Instruments}

1) Subjective pain

Subjective pain was measured using the visual analog scale (VAS). Injection pain shows side variation, and, given the very short duration of this pain, the VAS is an appropriate means of quickly measuring pain [20]. The VAS used to assess subjective pain had a $10-\mathrm{cm}$ horizontal line with ratings ranging from 0 (no pain) on the left end to 10 (severe pain) on the right end. Study participants themselves marked their pain level on the line, and higher scores indicated more severe pain.

2) Objective pain

To measure objective pain, the objective pain behavior checklist developed by Park [21] was employed in this study. This checklist consisted of 10 question items on facial expression, 8 on voice change, and 11 on perspiration level. According to the pain level, each question was scored on a scale of 0 to 4 . The objective pain scores ranged between 0 and 45, and higher scores indicated more severe pain.

3) Subjective anxiety

The participants' subjective anxiety was measured using the VAS with a $10-\mathrm{cm}$ horizontal line with scores ranging from 0 (no anxiety) on the left end to 10 (serious anxiety) on the right end. The participants themselves mark their anxiety level on the line, and higher scores indicated greater anxiety.

4) Anxiety status

To measure the patients' anxiety status, this study used only those items modified and improved by Lee [22] from among the 6 items (anxiety, depression, anger, vigor, fatigue, and confusion) in the profile of mood states (POMS) anxiety scale developed by McNair et al. This study instrument included a 5-point scale for each of the 9 question items on tension, instability, agitation, embarrassment, comfort, unpleasantness, dither, nervousness, and apprehension. The ratings on the 5-point scale were as follows: 0, "no at all"; 1, "a little bit"; 2, "fair"; 3, "much"; and 4, "very much." The higher the score, the stronger the anxiety was. The reliability of this instrument was found to be Cronbach's $\alpha=.87$ in Lee's study [22], but it was Cronbach's $\alpha=.72$ in the present study.

\subsection{Experimental Process}

1) Lidocaine spraying

A previous study found that lidocaine spraying produced a stronger anesthetic effect when performed 10 minutes rather than 5 minutes before venipuncture; the same study also found no difference in the anesthetic effect when spraying was performed between 10 minutes and 20 minutes. Therefore, in this study, lidocaine spraying was performed 10 minutes before venipuncture. In the previous study, the spraying frequency was set as three times per patient and no effect of spraying frequency was found on the extent of surface anesthesia. Therefore, in this research $10 \%$ lidocaine was sprayed three consecutive times on the venipuncture area [23].

2) Physiological saline spraying

Physiological saline was sprayed on the venipuncture area of the patients in the control group in the same manner as that for the experimental group. 


\subsection{Data Collection Process}

This study was conducted after receiving an approval from the institutional review board (IRB), which included a researcher involved in this study. The data were collected from 60 participants from February through March 2015. Of them, two decided to drop out in the middle of the process after two rounds of intravenous injections and three others refused to participate in this study. Finally, 55 participants (28 in the experimental group and 27 in the control group) were included in the study.

(1) A research assistant delivered the lidocaine or physiological saline in spray containers marked number 1 or 2 , without letting the researcher known what was inside each container (i.e., the researchers were blinded).

(2) The study participants threw a dice, and those who got an odd number received spray number 1 and those who got an even number received spray number 2.

(3) The participants were surveyed before venipuncture about their general characteristics, subjective anxiety, and anxiety status.

(4) The venipuncture area was marked on the skin of each participant, and the nebulizer with the number they had selected was sprayed three consecutive times on the marked area.

(5) After 10 minutes, venipuncture was performed on all of the participants with an $18 \mathrm{G}$ angio-catheter.

(6) The participants were surveyed immediately after the procedure to measure their subjective and objective pain levels, subjective anxiety, and anxiety status.

(7) To improve internal validity, Researcher A measured the participants' facial expression and Researcher B measured their voice change and perspiration level.

(8) The research assistant interchanged the contents of the spray containers (either lidocaine or physiological saline) once a week without the knowledge of the researchers.

\subsection{Data Analysis}

The data collected in this study were analyzed using IBM SPSS Statistics for Windows Version 21.0 (IBM Corp., Armonk, NY). The $\mathrm{X}^{2}$ test and t-test were implemented to investigate the general characteristics of the experimental group and control group, as well as the homogeneity of the variables. Pain was analyzed using the t-test, and anxiety was analyzed using ANCOVA.

\section{Study Results}

\subsection{General Characteristics of Study Participants and Homogeneity Test}

This study examined 55 participating subjects. Their general characteristics are shown in Table 1 . Of the 55 subjects, $28(50.9 \%)$ were in the experimental group and $27(49.1 \%)$ in the control group. Between the two groups, statistical homogeneity was found in terms of sex, age, intravenous injection experience, subjective anxiety, anxiety status, blood pressure, and heart rate. 
Table 1. General Characteristics of Participants

\begin{tabular}{|c|c|c|c|c|c|}
\hline & & & & & $(\mathrm{N}=55)$ \\
\hline & & $\begin{array}{l}\text { Experimental } \\
\text { group } \\
(\mathrm{n}=28)\end{array}$ & $\begin{array}{l}\text { Control } \\
\text { group } \\
(\mathrm{n}=27)\end{array}$ & & \\
\hline Characteristics & Categories & $\begin{array}{c}\mathrm{n}(\%) \\
\text { or } \\
\mathrm{M} \pm \mathrm{SD}\end{array}$ & $\begin{array}{c}\mathrm{n}(\%) \\
\text { or } \\
\mathrm{M} \pm \mathrm{SD}\end{array}$ & $\mathrm{X}^{2} / t$ & $p$ \\
\hline Age & $30-39$ & $5(17.9)$ & $2(7.4)$ & 3.20 & $.368 \dagger$ \\
\hline & $40-49$ & $5(17.9)$ & $2(7.4)$ & & \\
\hline & $50-59$ & $9(32.1)$ & $10(37.0)$ & & \\
\hline & 60 이상 & $9(32.1)$ & $13(48.1)$ & & \\
\hline Gender & Male & 26(92.9) & $26(96.3)$ & 0.32 & $.514 \dagger$ \\
\hline & Female & $2(7.1)$ & $1(3.7)$ & & \\
\hline Intravenous & Yes & $20(71.4)$ & $22(81.5)$ & 0.77 & .380 \\
\hline $\begin{array}{l}\text { lnjection } \\
\text { experience }\end{array}$ & No & $8(28.6)$ & $5(18.5)$ & & \\
\hline $\begin{array}{l}\text { Subjective } \\
\text { anxiety } \\
\text { (score) }\end{array}$ & & $1.25 \pm 1.89$ & $0.81 \pm 1.52$ & 0.95 & .347 \\
\hline $\begin{array}{l}\text { Anxiety status } \\
\text { (score) }\end{array}$ & & $4.43 \pm 2.69$ & $4.85 \pm 3.37$ & $\begin{array}{r}- \\
0.52\end{array}$ & .608 \\
\hline $\begin{array}{l}\text { Blood pressure } \\
(\mathrm{mmHg})\end{array}$ & Systolic & $123.79 \pm 14.85$ & $\begin{array}{c}132.04 \pm 20.0 \\
3\end{array}$ & 1.74 & .088 \\
\hline & Diastolic & $72.75 \pm 10.16$ & $76.81 \pm 14.82$ & 1.19 & .239 \\
\hline $\begin{array}{l}\text { Heart rate } \\
\text { (rate/1 min) }\end{array}$ & & $67.54 \pm 11.33$ & $64.82 \pm 11.31$ & $1 . \overline{4}$ & .146 \\
\hline
\end{tabular}

$\dagger$ Fisher's exact test

\subsection{Effect of Lidocaine Spraying on Intravenous Injection Pain}

Subjective pain level was significantly lower in the experimental group (1.79 \pm 1.79) than in the control group $(3.48 \pm 2.53)(\mathrm{t}=-2.86, p=.006)$. Accordingly, lidocaine spraying was found effective in reducing subjective pain.

Objective pain was also significantly lower in the experimental group (1.07 \pm $0.86)$ than in the control group $(2.15 \pm 1.20)(\mathrm{t}=-3.82, p<.001)$. Thus, lidocaine spraying was also found effective in reducing objective pain $\langle$ Table 2$\rangle$.

Among the sub-dimensions of objective pain, facial expressions were significantly fewer in the experimental group $(1.00 \pm 0.72)$ than in the control group $(1.89 \pm 1.09)(\mathrm{t}=-3.56, p<.001)$. Voice change and perspiration level showed no statistically significant intergroup differences. 
Table 2. Effect of Lidocaine Spraying on Pain

\begin{tabular}{|c|c|c|c|c|c|}
\hline \multirow{2}{*}{\multicolumn{2}{|c|}{ Variables }} & $\begin{array}{l}\text { Experimental } \\
\text { group } \\
(\mathrm{n}=28)\end{array}$ & $\begin{array}{l}\text { Control } \\
\text { group } \\
(\mathrm{n}=27)\end{array}$ & \multirow[t]{2}{*}{$\mathrm{t}$} & \multirow[t]{2}{*}{$p$} \\
\hline & & $\mathrm{M} \pm \mathrm{SD}$ & $\mathrm{M} \pm \mathrm{SD}$ & & \\
\hline \multicolumn{2}{|c|}{$\begin{array}{l}\text { Subjective pain } \\
\text { (score) }\end{array}$} & $1.79 \pm 1.79$ & $3.48 \pm 2.53$ & -2.86 & .006 \\
\hline \multirow{4}{*}{$\begin{array}{l}\text { Objective } \\
\text { pain } \\
\text { (score) }\end{array}$} & $\begin{array}{l}\text { Facial } \\
\text { expression }\end{array}$ & $1.00 \pm 0.72$ & $1.89 \pm 1.09$ & -3.56 & .001 \\
\hline & Voice change & $0.07 \pm 0.26$ & $0.22 \pm 0.80$ & -0.95 & .349 \\
\hline & $\begin{array}{l}\text { Perspiration } \\
\text { level }\end{array}$ & $0.00 \pm 0.00$ & $0.00 \pm 0.00$ & NA & NA \\
\hline & Total & $1.07 \pm 0.86$ & $2.15 \pm 1.20$ & -3.82 & $<.001$ \\
\hline
\end{tabular}

NA; Not applicable

\subsection{Effect of Lidocaine Spraying on Venipuncture Anxiety}

The ANCOVA was implemented to examine the effect of lidocaine spraying on post-test subjective anxiety in both groups. The results indicated that subjective anxiety was significantly lower in the experimental group $(1.11 \pm 1.56)$ than in the control group $(1.78 \pm 1.85)(\mathrm{F}=4.36, p=.042)$. Thus, in venipuncture, lidocaine spraying was found to have an effect on reducing the patients' subjective anxiety (Table 3).

Table 3. Effect of Lidocaine Spraying on Anxiety

\begin{tabular}{lllll}
\hline & $\begin{array}{l}\text { Pre-test } \\
\mathrm{M} \pm \mathrm{SD}\end{array}$ & $\begin{array}{l}\text { Post-test } \\
\mathrm{M} \pm \mathrm{SD}\end{array}$ & $\mathrm{F}^{*}$ & $p^{*}$ \\
\cline { 1 - 1 } $\begin{array}{l}\text { Experimental group } \\
(\mathrm{n}=28)\end{array}$ & $1.25 \pm 1.86$ & $1.11 \pm 1.56$ & & \\
\cline { 1 - 2 } $\begin{array}{l}\text { Control group } \\
(\mathrm{n}=27)\end{array}$ & $.81 \pm 1.52$ & $1.78 \pm 1.85$ & & .042 \\
\hline * ANCOVA & & & & \\
\hline
\end{tabular}

\subsection{Effect of Lidocaine Spraying on Anxiety Status}

The ANCOVA was implemented to examine the effect of lidocaine spraying on the post-test anxiety status of both groups. Anxiety status was significantly lower in the experimental group $(3.79 \pm 1.42)$ than in the control group $(5.65 \pm 4.10)(\mathrm{F}=$ $5.02, p=.028$ ). Thus, in venipuncture, lidocaine spraying was found to have an effect on easing the patients' anxiety status (Table 4). 
Table 4. Effect of Lidocaine Spraying on Anxiety Status

\begin{tabular}{lllll}
\hline & $\begin{array}{l}\text { Pre-test } \\
\mathrm{M} \pm \mathrm{SD}\end{array}$ & $\begin{array}{l}\text { Post-test } \\
\mathrm{M} \pm \mathrm{SD}\end{array}$ & $\mathrm{F}^{*}$ & $p^{*}$ \\
\cline { 1 - 1 } $\begin{array}{l}\text { Experimental group } \\
(\mathrm{n}=28)\end{array}$ & $4.43 \pm 2.67$ & $3.79 \pm 1.42$ & & \\
\cline { 1 - 2 } $\begin{array}{l}\text { Control group } \\
(\mathrm{n}=27)\end{array}$ & $4.85 \pm 3.37$ & $5.65 \pm 4.10$ & & .028 \\
\hline
\end{tabular}

* ANCOVA

\section{Discussion}

This study investigated the subjective and objective pain levels to examine the effect of lidocaine spraying on the intravenous injection pain of patients receiving contrast medium injection. The results showed that the experimental group that received lidocaine spraying had significantly lower subjective pain $(\mathrm{t}=-2.86, p=$ $.006)$ and objective pain $(\mathrm{t}=-3.82, p<.001)$ than did the control group. This finding is consistent with that of the study in which the EMLA cream was applied to patients undergoing venipuncture; the subjective pain level in the experimental group (2.75) was significantly lower than that in the control group (4.16) and the objective pain was also significantly lower in the experimental group [24]. The finding is also consistent with that of another study on intravenous injection pain experienced by patients in emergency rooms, which showed that $9.6 \%$ lidocaine could potentially ease subjective pain [25].

Intravenous injection is one of the most common invasive techniques, and it causes pain and anxiety in patients. Thus, diverse local anesthetic drugs, which are applied onto the patients' skin, have been developed to ease their pain. Lidocaine, in particular, is a major drug used for local anesthesia because of its quick effect, lack of local stimulation, and longer duration of action. Therefore, lidocaine has been used in infiltration anesthesia, peripheral nerve block, spinal and epidural anesthesia, etc. [25, 26]. Kanai [18] reported that in venipuncture, $8 \%$ lidocaine spraying and lidocaine patch application showed similar anesthetic effects, and spraying was quicker than patch application in producing a local anesthetic effect. These findings suggest that spraying a lidocaine containing drug is very effective in alleviating a patient's pain.

This study examined the effect of lidocaine spraying on the anxiety of patients receiving contrast medium injection during venipuncture. Lidocaine spraying was found effective in easing their anxiety. According to Song [15], 10\% lidocaine spraying was more effective than aromatic hand massage at lowering the anxiety of patients with arteriovenous aneurysm undergoing blood dialysis. Lidocaine ointment application was also effective in easing the anxiety of patients undergoing blood dialysis. Rather than $2 \%$ lidocaine, $5 \%$ lidocaine was found more effective in reducing anxiety [18], similar to the findings of the present study. However, Chung's study [27] applied a local anesthetic drug for 1 hour to ease pain and anxiety in children undergoing venipuncture. They measured the effectiveness of the drug and found that it had an effect on pain reduction but not on anxiety reduction. This suggested that the pain- and anxiety-lowering effect of lidocaine could be attributed to patient psychology rather than actual local drug action.

In contrast to the above findings, a previous study [23] found that a $2 \%$ lidocaine intradermal injection or $10 \%$ lidocaine spraying had no effect on pain or anxiety 
reduction in patients with arteriovenous aneurysm undergoing blood dialysis. Nevertheless, $35.3 \%$ of their study participants showed satisfaction with the spraying method, and 58.8\% wished to reuse it. The finding is not consistent with the findings of the present study in which lidocaine spraying helped ease anxiety. Further studies including more anxiety-related variables and more participants are necessary to explain such contrasting results.

\section{Conclusions}

With the frequent use of CT and MRI in clinical settings, the use of contrast media has also increased. Accordingly, 18 20G injection needles have become indispensable for the procedure. Considering the invasive nature of these procedures, nurses are required to provide appropriate intervention to relieve the patients' anxiety and pain. In such situations, lidocaine spraying can be a novel nursing intervention for alleviation of pain, discomfort, and anxiety in patients undergoing venipuncture. Nevertheless, additional large-scale studies in diverse settings will be necessary to confirm these results.

\section{References}

[1] D. C. Kweon, S. M. Hong and B. Park, "Comparison of Time Analysis on the Film Based System Versus PACS in the CT Scanning", Journal of the Korean Institute of Industrial Engineers, vol. 15, no. 4, (2002), pp. 439-443.

[2] G. Castedine, "New initiative to provide evidence-based IV care", British Journal of Nursing, vol. 11, no. 20, (2002), pp. 1351.

[3] A. F. Jacoban and E. H. Winslow, "Variables influencing intravenous catheter insertion difficulty and failure; An analysis of 339 intravenous catheter insertion", Heart Lung, vol. 34, no. 5, (2005), pp. 345359.

[4] D. C. Kweon and K. J. Jang, "Comparison Study with Pain Assessment Tools of Pain Measurement in Children for Intravenous Catheter Placement in the Computed Tomography", The Korea Contents Society, vol.8, no. 2, (2008), pp. 205-212.

[5] J. S. Park, "The Effect of Cutaneous Stimulation and Distraciton on IV Injection Pain of Chemotherapy Patients", Journal of Korean Academy of Nursing, vol. 28, no. 2, (1998), pp. 303-318.

[6] B. Barnhill, M. D. Holbert, N. M. Jacken \& R. Erickson, "Using pressure to decrease the pain of intra muscular injections", J. of Pain \& Symptiom Management, vol. 12, no. 1, (1996), pp. 52-58.

[7] H. J. Kim, S. K. Chaung, K. H. Kim and K. A. Kang, "The Relationship of Pain, Depression and Anxiety Which Patients Recognize on Intravenous Injection: Focus on Pain Relating Factors,” The Korean Journal of Fundamentals of Nursing, vol. 14, no. 3, (2007), pp. 306-314

[8] Y. S. Byun, J. S. Kang, A. K. Kim, J. H. Kim, J. A. Park and H. J. Park, "Fundamental nursing", Gyechuk Munwhasa, Seoul, (2012).

[9] S. K. Min, "Modern psychiatry", Ilchokak, Seoul, (2015).

[10] K. J. Lee, "Psychiatric Mental Health Nursing", Sumunsa, Paju, (2015).

[11] K. S. Cho, H. K. Kim, S. O. Park, Y. S. Bae and O. N. Hwang, "Adult Health Nursing", Hyunmunsa, Seoul, (2013).

[12] G. Çelik, O, Özbek, M. Yılmaz, I. Duman, S. Özbek and S. Apiliogullari, "Vapocoolant spray vs lidocaine/prilocaine cream for reducing the pain of venipuncture in hemodialysis patients: A randomized, placebo-controlled, crossover study”, International Journal of Medical Sciences, vol. 8, no. 7, (2011), pp. 623-627.

[13] E. H. Lee and S. E. Choi, "The Effects of Music Therapy by Self-Selected Music Listening on Terminal Cancer Patients' Affect and Stress by Pain Level", Korean journal of hospice and palliative care, vol.15, no.2, (2012), pp. 77-87.

[14] J. H. Kim, S. C. Lhim, S. W. Roh, S. J. Lee, Y. M. Ko, Y. O. Kim and Y. S. Shin, “ Effects of Sequential Application of Superficial Cold and Heat on Pain, Patient Satisfaction with Pain Control, Comfort Level and Subjective Response after Spine Surgery", The Korean journal of fundamentals of nursing, vol.23, no.2, (2016), pp. 184-193.

[15] J. M. Song and H. J. Park, "Effects of $10 \%$ Lidocaine Spray and Aroma Hand Massage on Pain, Anxiety, Blood Pressure, and Pulse During Arteriovenous Fistula Needling in Hemodialysis Patients", Korean J Rehabil Nurs, vol. 19, no.1, (2016), pp. 1-11.

[16] M. O. Shin, H. J. Park, E. J. Chang, Y. H. Suh, M. Y. Heo, M. K. Kim, M. L. Choi, M. J. Lee and Y. J. Kim, "A comparison of pain reducing effects of topical EMLA cream and subcutaneous lidocaine in hemodialysis patients", J Korean Fund Nurs, vol. 2, no. 1, (1995), pp. 37-44. 
[17] A. Schapowal, D. Berger, P. Klein and A. Suter, "Echinacea/Sage or Chlorhexidine/lidocaine for treating acute sore throats; a randomized double-blind trial", European Journal of medical Research, vol. 14, no. 9, (2009), pp. 406-412.

[18] A. Kanai, A. Suzuki, M, Kobayashi and S. Hoka, "Intranasal lidocaine 8\% spray for second-division trigeminal neuralgia,” British Journal of Anesthesia, vol. 97, no. 4, pp. (2006), pp. 559-563.

[19] F. Araimo, F. Bilotta, R. Caramia and G. Rosa, "Adrenaline through the endotracheal tube as an answer to severe bronchospasms caused by lidocaine”, Minerva Anestesiologica, vol. 76, no. 1, (2010), pp. 6365.

[20] J. Y. Choi, "A Comparison on the Degree of Pain according to Methods of Blood Sugar Test between DM Patients and Healthy Group," Journal of Korean Academy of Nursing, vol. 33, no.7, (2003), pp.928-935.

[21] J. S. Park, "The Effect of Cutaneus Stimulation on AV Fistula Puncture pain of Hemodialysis Patients," The Korean nurse, vol. 33, no.1, (1994), pp.37-51.

[22] M. S. Lee, "The Effect of Foot Massage on Patients with Post-operative Pain and Anxiety Following Abdominal Surger", Eulji University, Seongnam, (2009).

[23] J. K. Kim, M. S. Shim and K. H. Kim, "The Effect of topical EMLA Cream for venipuncture on Patients' Pain and Anxiety”, Journal of Korea Academia-Industrial cooperation Society, vol. 13, no. 9, (2012), pp. 4065-4072.

[24] Y. H. Lee and N. C. Kim: "The Comparison of Effects of 2\% Lidocaine Intradermal Injection and 10\% Lidocaine Spray for Pain and Anxiety Relief on Arteriovenous Fistula Puncture in Hemodialysis Patients," Journal of Korean Clinical Nursing Research, vol. 13, no. 2, (2007), pp.169-177.

[25] D. Park, J. Y. Ryu and G. C. Cho, "Original Articles: A Study to Evaluate the Efficacy of 9.6\% Lidocaine of Local Anesthesia for Pain Reduction of Venipuncture in the ED," Journal of the Korean Association of Traumatology, vol. 20, no. 2, (2007), pp. 115-118.

[26] M. S. Wang, S. O. Kim and H. C. Jeong, "A Comparative Study on Emotional Labor, Positive Resources, and Job Burnout of the Nurses Working in General Wards and Cancer Wards", Proceedings of the 10th International Workshop of Convergence Works II, Jeju, Korea, (2016) August 16-19.

[27] Y. S. Chung, K. W. Park and J. S. Kim, "Effects of EMLA Cream to Decrease Venipuncture Related Pain and Anxiety in Children", Child Health Nursing Research, vol. 12, no. 2, (2006), pp. 253-259. 
International Journal of Bio-Science and Bio-Technology Vol.8, No.6 (2016) 